\title{
The role of acidity in solid tumour growth and invasion
}

\author{
Kieran Smallbone $^{\mathrm{a}, *}$, David J. Gavaghan ${ }^{\mathrm{b}}$, Robert A. Gatenby ${ }^{\mathrm{c}}$, Philip K. Maini ${ }^{\mathrm{a}}$ \\ ${ }^{a}$ Centre for Mathematical Biology, Mathematical Institute, Oxford University, 24-29 St Giles', Oxford, OX1 3LB, UK \\ ${ }^{\mathrm{b}}$ Oxford University Computing Laboratory, Wolfson Building, Parks Road, Oxford, OX1 3QD, UK \\ ${ }^{\mathrm{c}}$ Department of Radiology, University of Arizona, 1501 N. Campbell Avenue, Tucson, Arizona 85724-5067, USA
}

Received 24 August 2004; received in revised form 1 February 2005; accepted 4 February 2005

Available online 16 March 2005

Communicated by Julian. H. Lewis

\begin{abstract}
Acidic $\mathrm{pH}$ is a common characteristic of human tumours. It has a significant impact on tumour progression and response to therapies. In this paper, we develop a simple model of three-dimensional tumour growth to examine the role of acidosis in the interaction between normal and tumour cell populations. Both vascular and avascular tumour dynamics are investigated, and a number of different behaviours are observed. Whilst an avascular tumour always proceeds to a benign steady state, a vascular tumour may display either benign or invasive dynamics, depending on the value of a critical parameter. Analysis of the model allows us to assess novel therapies directed towards changing the level of acidity within the tumour.
\end{abstract}

(C) 2005 Elsevier Ltd. All rights reserved.

Keywords: Tumour invasion; Glycolytic phenotype; Acidity; Mathematical modelling

\section{Introduction}

The tumour microenvironment is significantly different from that of normal tissue. Marked fluctuations can be seen in glucose, lactate, acidic $\mathrm{pH}$ and oxygen tensions. These variations have their roots in poor perfusion and metabolic changes. The chaotic vasculature of tumours creates an unbalanced blood supply and significant perfusion heterogeneities. As a consequence, many regions within tumours are found to be transiently or chronically hypoxic (oxygen deficient). Cells respond to periods of hypoxia by converting to anaerobic respiration, or glycolysis, which in turn produces lactic acid and brings about lower tissue $\mathrm{pH}$. However, the pioneering work of Warburg (1930) showed that tumour acidification can occur independently of hypoxia. The increased reliance on glycolysis to produce energy in many aggressive tumours occurs even in the presence of

\footnotetext{
*Corresponding author. Tel.: + 441865273518 ; fax: +441865273583

E-mail address: kieran.smallbone@new.ox.ac.uk (K. Smallbone).
}

sufficient oxygen (Vaupel et al., 1989; Warburg, 1930). This constitutive adoption of increased glycolysis, known as the glycolytic phenotype, is likely to have evolved in response to Darwinian selection dynamics in which phenotypes best suited to their microenvironment have a proliferative advantage over their non-transformed counterparts. Hence tumour acidification is an intrinsic property of both poor vasculature and altered tumour cell metabolism.

Cancer cell populations are extremely heterogeneous, displaying a wide range of genotypic and phenotypic differences (Fidler and Hart, 1982). For example, studies of clinical breast cancers have shown that every tumour cell exhibited a novel genotype (Kerangueven et al., 1997). As a result, no prototypic cancer cell can be defined. It is likely that several of the lethal phenotypic traits of cancer, such as invasion and metastasis, are not the direct result of genetic changes, but rather arise from the unique physiological environments of tumours. Tumour hypoxia and acidity, for example, significantly affect the treatment and progression of cancer. These effects can either be directly mediated by low $\mathrm{pH}$ or low 
$\mathrm{pO}_{2}$, or they can result from selective pressure that these parameters place upon cells in these hostile environments. Hypoxia and acidity are not simply phenomena of cancer growth, but may be in fact essential intermediates in the progression from benign to metastatic growth. Acidity, in particular, has been shown to have three clear effects on tumour phenotype: resistance to chemotherapy (Raghunand et al., 2001), increased mutation rate (Morita et al., 1992) and increased invasion (Martinez-Zaguilan et al., 1996).

Non-linear processes dominate the way in which tumour cells interact with their microenvironment. It is clear that the intuitive, verbal reasoning approaches favoured by many oncologists are insufficient to describe the resulting complex system dynamics. Nor can these approaches keep pace with the vast amounts of oncological data being published each year in response to the rapid technological advances in molecular biology. Rather, experience from other areas of science has taught us that quantitative methods are needed to develop comprehensive theoretical models for interpretation, organization and integration of this data (Gatenby and Maini, 2003). Once thought of as too simplistic to describe complex tumour phenomena, we now see that mathematical models, continuously revised by new information, can be used to guide experimental design and interpretation. Many of the recent mathematical models found in the literature focus on the growth of multicellular spheroids (MCSs): clusters of cancer cells grown in vitro to mimic the early stages of in vivo avascular tumour growth and to test the applicability of new cancer treatment strategies. MCSs have a welldefined structure, possessing a central core of necrotic cells, with proliferating cells restricted to the outer rim of the tumour. Existing models of MCS and avascular tumour development (Byrne, 1999; Franks et al., 2005; McElwain and Morris, 1978), essentially extensions of the original models of Burton (1966) and Greenspan (1972), describe the evolution of the tumour outer boundary in response to vital nutrients (in particular oxygen) and growth factors. Using the assumption of spherical or cylindrical symmetry, these models give good qualitative agreement with experimental results, reproducing both the growth patterns and macroscopic heterogeneities typical of MCSs and avascular tumours.

In this paper, we derive a model, similar to that developed by Greenspan (1972), for examining the role played by acidity in tumour growth and invasion. The commonality of altered tumour metabolism, in particular the adoption of the glycolytic phenotype in most cancers, led Gatenby and Gawlinski (1996, 2003) to propose the acid-mediated tumour invasion hypothesis. The key idea is that the transformed tumour metabolism with increased use of glycolysis and acid secretion alters the microenvironment by substantially reducing tumour extracellular $\mathrm{pH}\left(\mathrm{pH}_{e}\right)$, usually by more than $0.5 \mathrm{pH}$ units. The $\mathrm{H}^{+}$ions produced by the tumour then diffuse along concentration gradients into the adjacent normal tissue. This acidification leads to death of normal cells due to activation of $\mathrm{p} 53$-dependent apoptosis pathways, as well as loss of function of critical $\mathrm{pH}$-sensitive genes. Tumour cells, however, are relatively resistant to acidic $\mathrm{pH}_{e}$, due to mutant $\mathrm{p} 53$ genes. Whilst normal cells die in environments with a persistent $\mathrm{pH}$ below about 7, tumour cells typically exhibit a maximum proliferation rate in a relatively acidic medium ( $\mathrm{pH}$ 6.8) (Casciari et al., 1992). As a result, the tumour edge can be seen as forming a travelling wave progressing into normal tissue, preceded by another travelling wave of increased microenvironmental acidity. Modelling this hypothesis on the macroscopic scale allows us to investigate the general tissue dynamics in both vascular and avascular tumour growth. In particular, for tumour cells displaying the glycolytic phenotype, we determine the critical parameters that cause the change, within our modelling framework, from a benign to invasive growth pattern, which in turn suggests new therapeutic regimes for counteracting this invasive growth.

\section{Model analysis}

Following previous models, we assume that the tumour acts as an incompressible fluid. As such, local changes in the cell population, caused by the birth or death of cells, give rise to internal pressure gradients that induce cellular motion and the expansion or contraction of the tumour colony. This expansive force is counterbalanced by cell-cell adhesion forces at the tumour periphery that maintain the tumour as a compact mass. Subsequent tumour growth is determined by the interaction between these expansive and restraining forces.

We model the tumour as a sphere and assume that spherical symmetry prevails at all times. Whilst this assumption is valid for early tumour and MCS growth, during later development tumours often become asymmetric. Moreover, it has been suggested that some measure of the irregularity of the tumour boundary may provide clinicians with useful prognostic information (Cross et al., 1994). However, under the assumption of spherical symmetry, the model remains analytically tractable and allows us to perform analysis of the general tissue dynamics in response to acid production. A schematic cross-sectional view of a tumour and its surrounding normal tissue is given in Fig. 1. Let $R_{2}$ denote the tumour radius and $R_{1}$ the radius of the necrotic core. We assume that $R_{1}<R<R_{2}$ is a viable region where the proliferating tumour cells exist in a spatially homogeneous state at their carrying capacity $K_{T}$. We further assume $R<R_{1}$ is a necrotic region, containing no viable cells, and that the necrotic debris continually disintegrates into simpler compounds that 


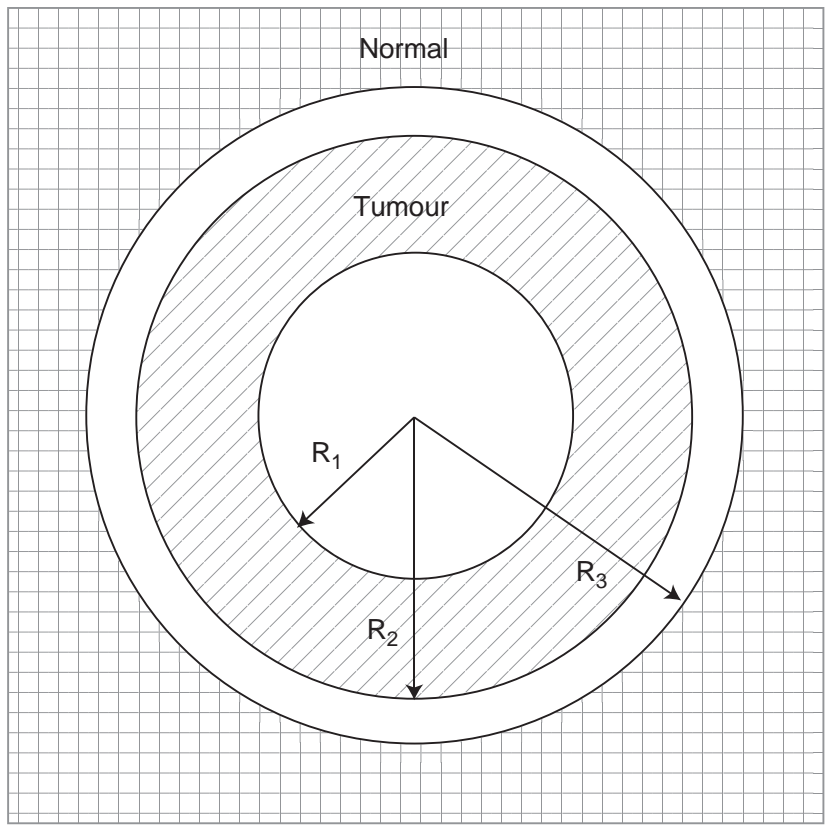

Fig. 1. Schematic cross-section of a tumour and its surrounding tissue, showing the central necrotic core, $R<R_{1}$, the layer of proliferating tumour cells $R_{1}<R<R_{2}$, the acellular gap separating normal and tumour cell fronts $R_{2}<R<R_{3}$, and the normal cells $R_{3}<R$.

are freely permeable through cell membranes. The cell volume lost in this way is replaced by cells pushed inward through adhesion or surface tension.

\subsection{Acid profile}

We consider first the distribution of acid generated by the tumour. Let $H$ denote the extracellular concentration of excess hydrogen ions. Here excess means above the normal level of $10^{-7.4} \mathrm{M}=\mathrm{pH}$ 7.4. It is assumed that there is a sharp acid threshold concentration $H_{T}$ above which tumour cells cannot survive. Similarly, normal cells die when this concentration $H$ rises above $H_{N}$. We assume $H_{N} \ll H_{T}$ to represent the relative resistance of tumour cells to extracellular acidity. As such, metabolically-produced acid can act both as a promoter or inhibitor of tumour growth. Diffusing into the normal tissue, the acid causes normal cell death which in turn allows the tumour to expand. Conversely, if acid is not removed from within the tumour sufficiently quickly, tumour cell death will occur. The interplay between these two mechanisms forms the heart of the model described below.

We assume that the evolution of $H$ can be described by a reaction-diffusion equation:

$$
\frac{\partial H}{\partial t}=F_{H}+D_{H} \nabla^{2} H
$$

where $D_{H}$ is the (assumed constant) acid diffusion coefficient and $F_{H}$ represents the combined rate of acid production and removal from the system.
Acid is produced by tumour cells as a result of their increased reliance on glycolysis and we assume that this occurs at a constant rate $r_{T}$ per unit volume. The primary mode for removal of acid from the system is through blood vessels and we assume that this occurs at a rate $r_{V}$ proportional to the local acid concentration. Note that the acid diffusion time-scale ( $\sim$ minutes) is much shorter than the tumour growth time-scale ( $\sim$ days). Hence, as the tumour grows, the acid quickly redistributes and reaches equilibrium. Following previous work, we assume that $H$ is in diffusive equilibrium at all times and set $\partial H / \partial t=0$ in the acid reactiondiffusion equation. Under these assumptions, and noting spherical symmetry, Eq. (1) becomes

$0=r_{T} T-r_{V} V H+\frac{D_{H}}{R^{2}} \frac{\mathrm{d}}{\mathrm{d} R}\left(R^{2} \frac{\mathrm{d} H}{\mathrm{~d} R}\right)$,

where $T$ denotes the viable tumour cell density and $V$ the vascular density.

We consider separately the acid profiles generated by vascular and avascular tumours. In the avascular case, we define $V=0$ for $R<R_{2}$ and $V=K_{V}$ elsewhere i.e. there is no vasculature within the tumour and the vasculature exists homogeneously at its normal level outside the tumour. Taking tumour cell density $T$ to be constant $\left(K_{T}\right)$ within the viable region $R_{1}<R<R_{2}$, and further taking $q=\sqrt{r_{V} K_{V} / D_{H}}$ and $H_{0}=r_{T} K_{T} / r_{V} K_{V}$, we may non-dimensionalize Eq. (2) with $r=q R$ and $h=$ $H / H_{0}$ to obtain:

$r^{2} h^{\prime \prime}+2 r h^{\prime}= \begin{cases}0 & 0<r<r_{1}, \\ -r^{2} & r_{1}<r<r_{2}, \\ r^{2} h & r_{2}<r,\end{cases}$

where the primes denote the derivative with respect to $r$.

Previous models of tumour growth have made the assumption that the nutrients and other factors determining tumour growth are constant outside the tumour tissue i.e. for any growth factor $g, g(r)=g_{\infty}$ for $r>r_{2}$. In the case of acid, however, this would be inconsistent with the data of Martin and Jain (1994). Reporting in vivo extracellular $\mathrm{pH}$ profiles for $\mathrm{VX} 2$ rabbit carcinoma, they demonstrate a smooth $\mathrm{pH}$ gradient extending from the tumour edge into the peritumoural normal tissue. Instead, we assume here that $\lim _{r \rightarrow \infty} h(r)=0$ i.e. that there is no excess acidity a long distance from the tumour. Assuming further that $h$ and its derivative are continuous at $r_{1}$ and $r_{2}$, and that $\lim _{r \rightarrow 0} h(r)$ is finite, Eq. (3) has solution

$h_{a}(r)= \begin{cases}k_{1} & 0<r<r_{1}, \\ k_{2}-k_{3} \frac{1}{r}-\frac{1}{6} r^{2} & r_{1}<r<r_{2}, \\ k_{4} \frac{1}{r} \mathrm{e}^{-r} & r_{2}<r,\end{cases}$ 
where the constants $k_{i}$ are given by

$k_{1}=\frac{2 r_{1}^{3}+3 r_{2}^{2}+r_{2}^{3}}{6\left(r_{2}+1\right)}-\frac{r_{1}^{2}}{2}$,

$k_{2}=\frac{2 r_{1}^{3}+3 r_{2}^{2}+r_{2}^{3}}{6\left(r_{2}+1\right)}$

$k_{3}=\frac{r_{1}^{3}}{3}$,

$k_{4}=\frac{\mathrm{e}^{r_{2}}\left(r_{2}^{3}-r_{1}^{3}\right)}{3\left(r_{2}+1\right)}$.

Returning to Eq. (2), we also calculate the predicted acid profile for a vascularized tumour. In this case we define $V=0$ for $r<r_{1}$ and $V=K_{V}$ elsewhere i.e. the vasculature exists in a spatially homogeneous state at its normal level throughout the tumour cell population. For simplicity, we neglect the poor efficiency ('leakiness') and heterogeneities generally found in tumour vasculature, considering only the extreme case where the tumour is fully vascularized. Moreover, we assume there is no vasculature within the necrotic core. Non-dimensionalizing as before, we find

$r^{2} h^{\prime \prime}+2 r h^{\prime}= \begin{cases}0 & 0<r<r_{1}, \\ r^{2}(h-1) & r_{1}<r<r_{2}, \\ r^{2} h & r_{2}<r\end{cases}$

with solution

$h_{v}(r)= \begin{cases}k_{1} & 0<r<r_{1}, \\ 1-k_{2} \frac{1}{r} \mathrm{e}^{-r}-k_{3} \frac{1}{r} \mathrm{e}^{r} & r_{1}<r<r_{2}, \\ k_{4} \frac{1}{r} \mathrm{e}^{-r} & r_{2}<r,\end{cases}$

where

$k_{1}=1-\frac{\mathrm{e}^{r_{1}-r_{2}}\left(r_{2}+1\right)}{r_{1}+1}$,

$k_{2}=\frac{\mathrm{e}^{2 r_{1}-r_{2}}\left(r_{1}-1\right)\left(r_{2}+1\right)}{2\left(r_{1}+1\right)}$,

$k_{3}=\frac{\mathrm{e}^{-r_{2}}\left(r_{2}+1\right)}{2}$,

$k_{4}=\frac{\mathrm{e}^{r_{2}}\left(r_{2}+1\right)}{2}-\frac{\mathrm{e}^{2 r_{1}-r_{2}}\left(r_{1}-1\right)\left(r_{2}+1\right)}{2\left(r_{1}+1\right)}$.

An example of this predicted acid profile can be seen in Fig. 2, with $r_{1}=1$ and $r_{2}=1.5$, and a comparison with the predicted avascular profile. Given experimentally determined parameter estimates of $q=0.47 \mathrm{~mm}^{-1}$ and $H_{0}=1.0 \times 10^{-5} \mathrm{M} \equiv \mathrm{pH} 5.0$ (Gatenby and Gawlinski, 1996; Martin and Jain, 1994), this corresponds to a tumour of radius $R_{2} \approx 3 \mathrm{~mm}$, with necrotic core radius $R_{1} \approx 2 \mathrm{~mm}$. Notice that the model predicts acidity for an avascular tumour to be higher than that for a vascular tumour, when both tumours produce acid at the same rate. This is to be expected given that there is no acid removal within the tumour in the avascular case. Note,

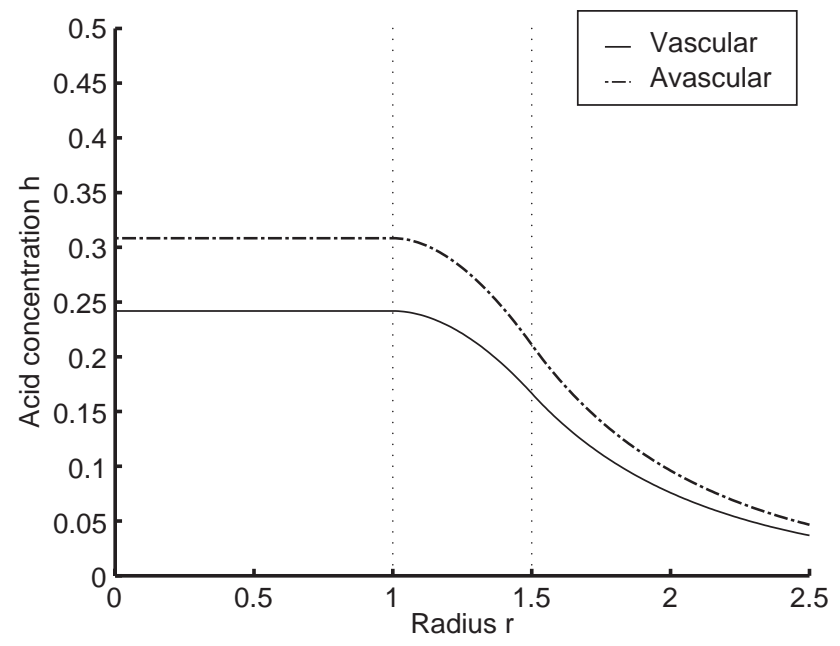

Fig. 2. (From Eqs. (4) and (7).) Predicated acid profile in the vascular case with $r_{1}=1$ and $r_{2}=1.5$, and a comparison with the corresponding avascular profile.

however, that due to an increased reliance on glycolysis, vascular tumours are often found to be more acidic than their avascular counterparts. In the model, this is represented by a higher value of $H_{0}$.

\subsection{Necrotic core development}

Previous models of tumour growth have assumed tumour necrosis occurs as a result of insufficient nutrient supply. In this paper, we focus on the effect of acidmediated tumour necrosis on the system. Assuming that high acidity is the sole cause of necrosis in the tumour allows us to calculate the radius of the necrotic core $r_{1}$ in terms of the tumour outer radius $r_{2}$.

In the avascular case and in the absence of a necrotic core (i.e. when $r_{1}=0$ ), from Eq. (4) we have:

$h_{a}(0)=\frac{r_{2}^{2}\left(r_{2}+3\right)}{6\left(r_{2}+1\right)} \longrightarrow \infty \quad$ as $r_{2} \rightarrow \infty$.

Thus at some critical value of $r_{2}, h_{a}(0)>h_{T}$ and the cells at the centre of the tumour will become necrotic. The critical radius $\hat{r}_{2}$ at which the necrotic core develops can be found by solving $h_{a}(0)=h_{T}$, with $r_{1}=0$ :

$c_{a}\left(\hat{r}_{2}\right)=\hat{r}_{2}^{3}+3 \hat{r}_{2}^{2}-6 h_{T} \hat{r}_{2}-6 h_{T}=0$.

By Descartes' rule of signs, this cubic has exactly one positive real root, and this is given by

$\hat{r}_{2}=2 \sqrt{2 h_{T}+1} \cos \left[\frac{1}{3} \arccos \left(-\left(2 h_{T}+1\right)^{-\frac{3}{2}}\right)\right]-1$,

where we choose $\arccos :[-1,1] \rightarrow[0, \pi]$. Taking the threshold for tumour death due to acidity to be $h_{T}=$ 0.1 , corresponding to $H_{T} \equiv \mathrm{pH} 6$ (Dairkee et al., 1995), we find that necrosis due to acidity first occurs at $\hat{r}_{2}=$ $0.51\left(\hat{R}_{2} \approx 1 \mathrm{~mm}\right)$. 
If $r_{2}>\hat{r}_{2}$, then a necrotic core exists, and its radius $r_{1}$ can be found by noting that the acid concentration at the boundary of the necrotic core will be $h_{a}\left(r_{1}\right)=h_{T}$ :

$2 r_{1}^{3}-3\left(r_{2}+1\right) r_{1}^{2}+c_{a}\left(r_{2}\right)=0$.

In this case we have exactly two positive real roots. Choosing the root of this cubic satisfying $0<r_{1}<r_{2}$, we find

$r_{1}=\frac{r_{2}+1}{2}\left[1+2 \cos \left[\frac{1}{3}\left(\pi+\arccos \left(\frac{2 c_{a}\left(r_{2}\right)}{\left(r_{2}+1\right)^{3}}-1\right)\right)\right]\right]$,

where again $\arccos :[-1,1] \rightarrow[0, \pi]$.

From Eq. (12):

$\lim _{r_{2} \rightarrow \infty}\left(r_{2}-r_{1}\right)=\sqrt{2 h_{T}+1}-1$

and hence

$\frac{r_{1}}{r_{2}} \longrightarrow 1, \quad \operatorname{Vol}\left(r_{1}, r_{2}\right)=\frac{4 \pi}{3}\left(r_{2}^{3}-r_{1}^{3}\right) \longrightarrow \infty \quad$ as $r_{2} \rightarrow \infty$.

This means that a large tumour will be mostly comprised of the necrotic core, with the layer of viable cells limited to a thin region at the tumour edge. Nonetheless, the total number of viable cells will continue to increase as the tumour grows.

Turning now to the vascular case, in the absence of a necrotic core we have, from Eq. (7):

$h_{v}(0)=1-\mathrm{e}^{-r_{2}}\left(r_{2}+1\right) \longrightarrow 1 \quad$ as $r_{2} \rightarrow \infty$

and hence we see two distinct patterns of growth, depending on the sign of $h_{T}-1$. If $h_{T} \geqslant 1, h_{v}(0)<h_{T}$ for any value of $r_{2}$; the tumour vasculature removes the excess acid sufficiently quickly to avoid tumour cell death and no necrotic core will develop. If, however, $h_{T}<1$, at some value of $r_{2}, h_{v}(0) \geqslant h_{T}$ and a necrotic core will develop. This critical radius $\hat{r}_{2}$ can be found by solving $h_{v}(0)=h_{T}$, with $r_{1}=0$, leading to the equation

$c_{v}\left(\hat{r}_{2}\right)=\mathrm{e}^{-\hat{r}_{2}}\left(\hat{r}_{2}+1\right)+\left(h_{T}-1\right)=0$

with solutions

$\hat{r}_{2}=-1-W\left(\frac{h_{T}-1}{\mathrm{e}}\right)$.

Here $W$ denotes the multivalued Lambert $\mathrm{W}$ (or product $\log$ ) function - the inverse function of $f(W)=W \mathrm{e}^{W}$. Note that for $-1 / \mathrm{e} \leqslant x<0$, there are two possible real values of $W(x), W_{0}(x) \geqslant-1$ and $W_{-1}(x) \leqslant-1$ (Corless et al., 1996). As $\hat{r}_{2} \geqslant 0$, for $h_{T}<1$ we can define

$\hat{r}_{2}=-1-W_{-1}\left(\frac{h_{T}-1}{\mathrm{e}}\right)$.

Further, for $h_{T}<1$ and $r_{2}$ greater than this critical radius, we find $r_{1}$ by solving $h_{v}\left(r_{1}\right)=h_{T}$ :

$\mathrm{e}^{r_{1}-r_{2}}\left(r_{2}+1\right)+\left(h_{T}-1\right)\left(r_{1}+1\right)=0$ with solution

$r_{1}=-1-W_{-1}\left(\frac{\mathrm{e}^{-\left(r_{2}+1\right)}\left(r_{2}+1\right)}{h_{T}-1}\right)$.

From Eq. (20), we find

$\lim _{r_{2} \rightarrow \infty}\left(r_{2}-r_{1}\right)=-\log \left(1-h_{T}\right)$

and hence as in the avascular case, Eq. (15) holds.

\subsection{Tumour growth}

We consider now the growth dynamics of the tumour in the absence of normal cells. As such, we analyse the inhibitory effects of acidity on tumour growth, whilst neglecting the invasive dynamics arising through the destruction of normal tissue. The rate at which a tumour grows may be dependent on a large number of factors, such as nutrient supply, cellular density or internal pressure gradients. Here we make the simplifying assumption that the rate of change of tumour volume is entirely dependent on the tumour radius and the radius of the necrotic core:

$\frac{\mathrm{d}}{\mathrm{d} t}(\mathrm{Vol})=F\left(R_{1}, R_{2}\right)$

for some mitosis function $F$.

Greenspan (1972) makes the assumption that the necrotic cellular debris continually disintegrates into simpler chemical compounds at a rate proportional to the core volume. These compounds flow into the surrounding tissue and the cell volume lost in this way is replaced by cells pushed inward through surface tension forces. Moreover, the assumption is made that the rate of cellular proliferation is constant per unit volume in the viable region. Under these assumptions, Eq. (23) becomes

$\frac{\mathrm{d} R_{2}^{3}}{\mathrm{~d} t}=S\left(R_{2}^{3}-R_{1}^{3}\right)-L R_{1}^{3}$.

Taking $\tau=S t / 3$ and $r=q R$, we may non-dimensionalize the system to obtain

$r_{2}^{2} \frac{\mathrm{d} r_{2}}{\mathrm{~d} \tau}=r_{2}^{3}-\gamma^{3} r_{1}^{3}$,

where $\gamma=\sqrt[3]{L / S+1}$

Note that while there is no necrotic core (when $r_{1}=0$ ), the tumour radius will grow exponentially with

$r_{2}(\tau)=r_{2}(0) \mathrm{e}^{\tau}$

This corresponds to well-known experimental evidence that the early stages of solid tumour development follow a simple exponential growth pattern (Laird, 1964). In particular, in the case of a vascular tumour with $h_{T} \geqslant 1$, the model predicts that a necrotic core will never 
develop and thus the tumour will continue to grow exponentially into the surrounding tissue. For an avascular tumour or a vascular tumour with $h_{T}<1$, however, a different growth pattern is observed. From Eq. (15), we know that $r_{1} / r_{2} \rightarrow 1$ as $r_{2} \rightarrow \infty$. Assuming that, at time 0 , the tumour is small enough that there is no necrotic core (i.e. $r_{2}(0) \leqslant \hat{r}_{2}$ ), at some value of $r_{2}$ we will find $r_{1}=r_{2} / \gamma<r_{2}$. Then from Eq. (25), $\mathrm{d} r_{2} / \mathrm{d} \tau=0$, and a benign steady state is reached. In other words, we find that an avascular tumour will always have a benign growth pattern. A vascular tumour will either have a benign or invasive growth pattern dependent on the value of the critical parameter $h_{T}$.

The system is completely defined by Eq. (25) and Eq. (13) or Eq. (21), and relies only on the parameters $\gamma$, $h_{T}$ and the initial condition $r_{2}(0)$. Examples of the growth patterns observed are given in Fig. 3. In the avascular case (a), a two-phase growth pattern is observed. Initially, the tumour grows exponentially, without a necrotic core. At the critical time $\hat{\tau}$, a necrotic core begins to develop and the second phase of tumour growth begins. During this phase, we see very little change in tumour size. However, the necrotic core grows rapidly towards its equilibrium value. Note that $\gamma$ represents the equilibrium $r_{2}: r_{1}$ ratio. The corresponding vascular growth is very similar when $h_{T}<1$ (b) and may be contrasted to the invasive growth seen when $h_{T} \geqslant 1$ (c). In this final case, as $r_{2}$ becomes large, other limiting factors such as nutrient supply and immune response will have more impact on the tumour growth.

The time $\hat{\tau}$ at which we see the onset of necrosis can be found from Eq. (26), taking $r_{2}=\hat{r}_{2}$ :

$\hat{\tau}=\log \hat{r}_{2}-\log r_{2}(0)$.

Using parameters $h_{T}=0.1$ and $r_{2}(0)=0.1 \quad\left(R_{2}(0) \approx\right.$ $0.2 \mathrm{~mm}$ ), we find necrosis occurs at $\hat{\tau}=1.63$ and $\hat{\tau}=$ 1.67 in the avascular and vascular cases, respectively. The equilibrium size $\bar{r}_{2}$ may be found by noting that $\bar{r}_{2}=\gamma \bar{r}_{1}$. In the avascular case, using Eq. (12) we find

$\bar{r}_{2}=\frac{\gamma}{(\gamma-1)(\gamma+2)}\left[-(\gamma+1)+2 c_{1} \cos \left[\frac{1}{3} \arccos \left(-\frac{c_{2}}{c_{1}^{3}}\right)\right]\right]$,

where

$c_{1}=\sqrt{(\gamma+1)^{2}+2 h_{T} \gamma(\gamma+2)}$,

$c_{2}=(\gamma+1)^{3}+6 h_{T}(\gamma+2)$.

For the parameter set used in Fig. 3, we find $\bar{r}_{2}=0.75$, corresponding to a final radius of $\bar{R}_{2} \approx 1.6 \mathrm{~mm}$. For the vascular case, we use Eq. (20), again setting $\bar{r}_{2}=\gamma \bar{r}_{1}$. Solving this numerically, we find $\bar{r}_{2}=0.80$ correspond-

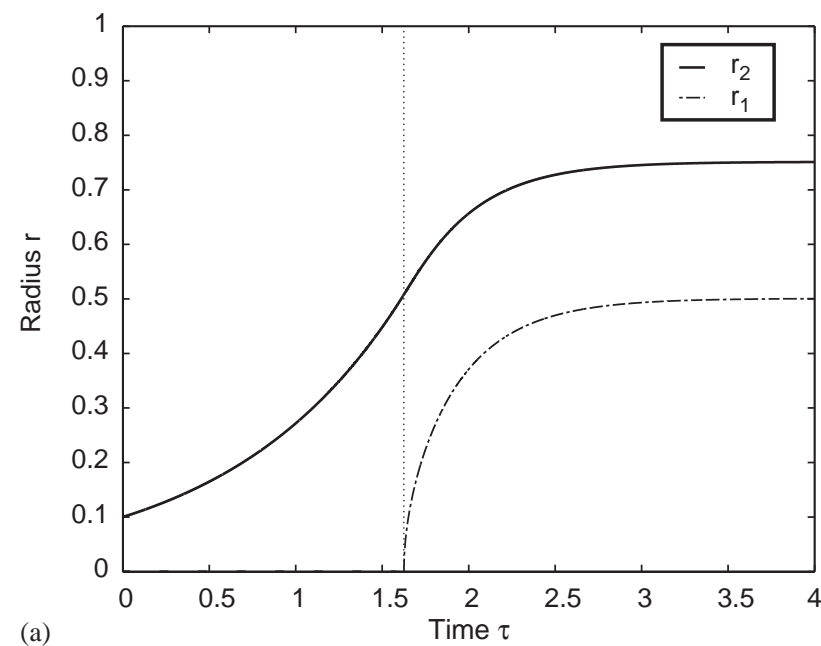

(a)
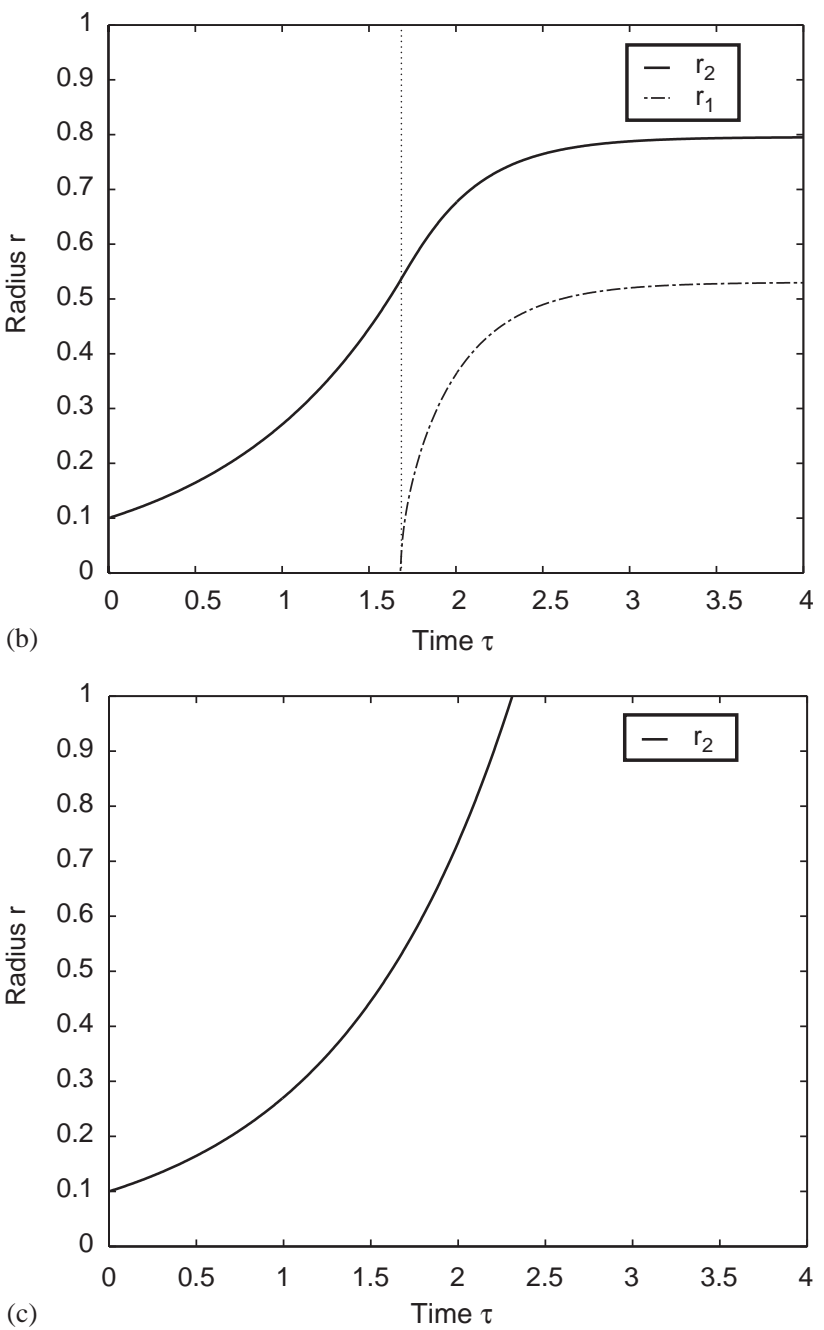

Fig. 3. (From Eqs. (13), (21) and (25).) Predicated (a) avascular and (b) vascular tumour growth with parameters $\gamma=3 / 2, h_{T}=0.1$ and $r_{2}(0)=0.1$. (c) Vascular growth with $h_{T}=1.5$ and $r_{2}(0)=0.1$.

ing to $\bar{R}_{2} \approx 1.7 \mathrm{~cm}$. These numbers demonstrate further the similarity between avascular and vascular growth when $h_{T}$ is small. 


\subsection{Normal tissue invasion}

We now move on to consider the effects of normal tissue on the system. Let $r_{3}$ denote the non-dimensionalized distance from the tumour centre to the normal tissue. Assume that initially the system has $r_{1}=0$ and $r_{3}=r_{2}$ i.e. the tumour is small enough that there is no necrotic core. Normal cells die if $h$ increases above a critical value $h_{N}=H_{N} / H_{0}$, where $h_{N} \ll h_{T}$.

In the vascular case, from Eq. (7):

$$
h_{v}\left(r_{2}\right)=\left(r_{2} \cosh r_{2}-\sinh r_{2}\right) \frac{\mathrm{e}^{-r_{2}}}{r_{2}} \quad\left(\text { as } r_{1}=0\right) \text {. }
$$

The normal tissue will recede and the tumour advance if and only if $h\left(r_{2}\right)>h_{N}$. Note that $h$ is an increasing function of $r_{2}$, and hence if $h\left(r_{2}(0)\right)>h_{N}$, then the tumour will grow unimpeded as was seen when normal tissue was neglected in the system.

In the avascular case, from Eq. (4):

$$
h_{a}\left(r_{2}\right)=\frac{r_{2}^{2}}{3\left(1+r_{2}\right)} \quad\left(\text { as } r_{1}=0\right) \text {. }
$$

Again the normal tissue will recede if and only if $h\left(r_{2}\right)>h_{N}$. Taking $h_{N}=0.01$, corresponding to $H_{N} \equiv$ $\mathrm{pH} 6.8$ (Dairkee, 1995), we find that in both the vascular and avascular cases, invasion will occur only if $r_{2} \geqslant 0.19$, equivalent to $R_{2} \approx 0.4 \mathrm{~mm}$.

If $h\left(r_{2}\right)>h_{N}$, then we can calculate $r_{3}$ through solution of the equation $h\left(r_{3}\right)=h_{N}$, i.e. $k_{4} \mathrm{e}^{-r_{3}} / r_{3}=h_{N}$ :

$r_{3}=W_{0}\left(\frac{k_{4}}{h_{N}}\right)$

where here we choose the principal value of the Lambert W-function as $k_{4}>0$ and $h>0$.

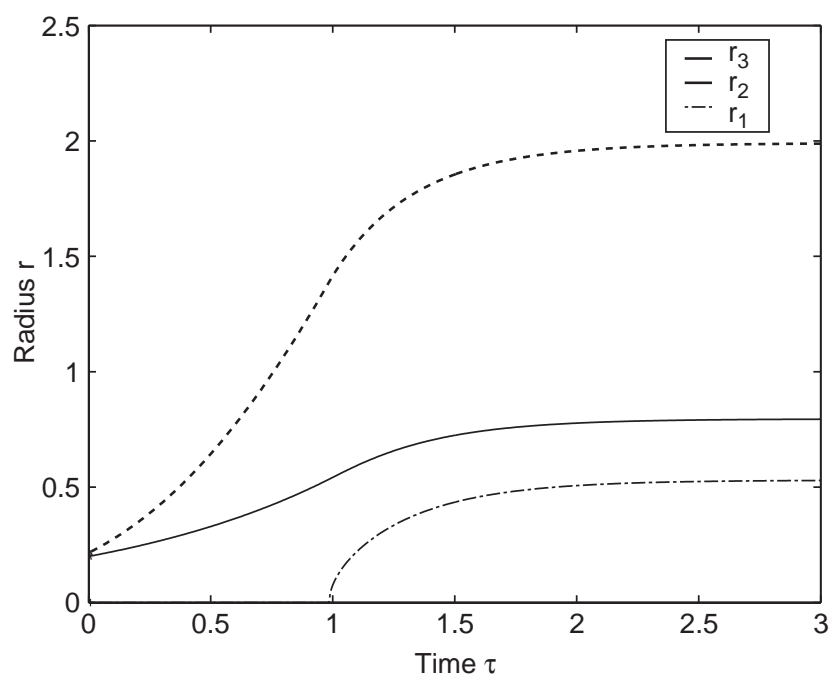

Fig. 4. (From Eq. (32).) Recession of normal tissue accompanying vascular tumour growth. Parameter values used are $h_{N}=0.01, \gamma=$ $3 / 2, h_{T}=0.1$ and $\gamma_{2}(0)=0.2$.
Fig. 4 shows normal tissue receding as the tumour grows. Notice the development of an acellular gap between the advancing tumour front and receding normal tissue, consistent with experimental observations (Gatenby and Gawlinski, 1996).

\section{Discussion}

In this paper, we have presented a mathematical study of both vascular and avascular tumour growth, where the invasion mechanism is the acidification of the microenvironment surrounding the tumour due to increased reliance on glycolysis. Utilising the vast difference between the time-scales of tumour growth and acid movement allows us to treat the tumour radius as a parameter in terms of which other variables are expressed. In particular, we determine the equilibrium acid profile and necrotic core radius as a function of the tumour radius.

The analysis predicts three regimes of tumour growth. If the rate of acid removal from the tumour is insufficient, we see exponential growth followed by auto-toxicity, resulting in a benign tumour. This is found always to occur in an avascular tumour, and it may also occur in a vascular tumour if the critical parameter $h_{T}<1$. Conversely, if $h_{T} \geqslant 1$, a vascular tumour displays sustained growth, and invades the whole of the normal tissue space. In both of these cases, the advancing tumour front is separated from the receding normal tissue by an acellular gap. Finally, if the tumour is sufficiently small, we see no growth as the microenvironmental acid perturbations are insufficient to induce normal cell death. Note, however, that for tumours of this size, inhomogeneities have more effect on the system and thus stochastic or cellular automaton (Patel et al., 2001) approaches may be more applicable than the mean-field type approach used here.

Within the model, three dominant factors determine tumour growth: acid production, acid removal due to tumoural and peritumoural vascularity, and cellular sensitivity to acid. In general, tumour growth is enhanced through increasing acid production to induce maximal toxicity in the adjacent normal tissue. However, in order to display sustained growth, the tumour must limit excess acid accumulation to avoid auto-toxicity. This balance may resolve itself in several ways. Tumour growth could be limited by cellular sensitivity: that is, the dominant populations within the tumour may retain significant sensitivity to acid-induced apoptosis. As such, tumour expansion is halted when the intratumoural $\mathrm{pH}_{e}$ is only modestly reduced. Tumour growth could also be limited if the vascularity is limited: the intratumoural hydrogen ions will accumulate sufficiently to create an acidic $\mathrm{pH}_{e}$ that halts proliferation. In these settings, tumour growth could be 
rapidly increased through adoption of the angiogenic phenotype or emergence of new populations with additional mutations that render them more resistant to acid-induced apoptosis. Finally, tumour growth could be limited by acid production: the tumour does not produce enough acid to create a peritumoural hydrogen ion gradient sufficient to kill the normal cells. In this case, emergence of phenotypes with higher glycolytic metabolism will result in invasive growth, consistent with findings that rate of glucose uptake correlates with more malignant behaviour (Kunkel et al., 2003).

The converse of each of the above scenarios suggests possible cancer treatment strategies. In general, the results favour tumour antiangiogenesis strategies, because decreased vascular density will reduce acid removal as well as nutrient supply. If the resulting decrease in $\mathrm{pH}_{e}$ exceeds the tolerance of tumour cells to local acidosis, the resulting apoptosis would halt tumour growth. Mathematically this is achieved through reducing $h_{T}=H_{T} r_{V} K_{V} / r_{T} K_{T}$ below the critical value of 1 . This parameter may also be reduced through the novel strategy of manipulating systemic $\mathrm{pH}$. A recent study demonstrated that patients with metastatic renal cancer benefit from cytoreductive nephrectomy (Gatenby et al., 2002). The authors propose that removal of functioning nephrons produces mild renal failure that is associated with systemic acidosis. This decrease in the serum $\mathrm{pH}$ will reduce acid removal, since diffusion of hydrogen ions from the tumour interstitium into blood vessels will be dependent on the concentration gradient across the vessel wall. The resulting decrease in intratumoural $\mathrm{pH}_{e}$ may again induce tumour auto-toxicity. However, both approaches above come with a cautionary note. Reduced acid removal will result in an increased peritumoural $\mathrm{pH}$ gradient, thus increasing degradation of normal tissue and thus potentially promoting tumour growth. As such, perhaps the most effective treatment suggested by the model is to poison the membrane pumps that transport hydrogen ions from the tumour intracellular to extracellular space (through drugs such as amiloride, for example). This would increase the tumour cell sensitivity to $\mathrm{pH}_{e}$, and furthermore decrease the peritumoural acid gradient.

The model's predictions may be compared to experimental results and clinical observations. The prediction of the presence and range of a $\mathrm{pH}$ gradient extending into the peritumoural normal tissue is consistent with the data of Martin and Jain (1994). We also demonstrate that whilst acidity correlates with increased tumour invasion (Martinez-Zaguilan et al., 1996), brief systemic acidosis may induce widespread tumour apoptosis and regression (Kelley et al., 2002). The most verifiable prediction is the development of an appreciable acellular gap separating the advancing tumour and receding normal tissue edges. Our analysis shows that the existence of such a gap is dependent only on tumour size and acid production rates and thus should be apparent in a wide range of cancer types. In a study performed on human head and neck carcinoma, this acellular gap was observed in $67 \%$ of cases (Gatenby and Gawlinski, 1996). It should be noted, however, that tumours use a variety of mechanisms to invade normal tissue. As such, they may create insufficient acid perturbations to induce an acellular gap, but nonetheless continue to grow. In these cases, additional mechanisms to tissue acidification must be considered.

It is clear that tumour growth is dependent on the complex interactive dynamics of many different factors, including the supply of nutrients and growth factors and the specific mutations displayed by the tumour population. This growth is further complicated by any inhomogeneities found within the tumour. Using simplifying assumptions, we have shown here that increased tumour acid production alone, almost universally observed in clinical cancers, is sufficient to explain both benign and invasive growth. As such, acidity may play a dominant role in tumour progression. Critical parameters in the transition from premalignant to malignant morphology include acquisition of angiogenesis, increased glucose utilization and loss of critical $\mathrm{pH}$-sensitive genes, all observed in human tumours. Various therapeutic strategies are suggested to inhibit tumour growth. In particular, the model suggests the counter-intuitive approach of increasing further tumour acidity, in order to induce auto-toxicity. Experimental results further verifying this observation would be of considerable interest.

\section{Acknowledgements}

KS and DJG are pleased to acknowledge the support of the Engineering and Physical Sciences Research Council through the Oxford University Life Sciences Doctoral Training Centre which has allowed them to undertake this research (GR/R96149/01).

\section{References}

Burton, A.C., 1966. Rate of growth of solid tumours as a problem of diffusion. Growth 30, 157-176.

Byrne, H.M., 1999. A weakly nonlinear analysis of a model of avascular solid tumour growth. J. Math. Biol. 39, 59-89.

Casciari, J.J., Sotirchos, S.V., Sutherland, R.M., 1992. Variations in tumor cell growth rates and metabolism with oxygen concentration, glucose concentration, and extracellular $\mathrm{pH}$. J. Cell Physiol. 151, 386-394.

Corless, R.M., Gonnet, G.H., Hare, D.E.G., Jeffrey, D.J., Knuth, D.E., 1996. On the Lambert W function. Adv. Comp. Math. 5, 329-359. 
Cross, S.S., Bury, J.P., Silcocks, P.B., Stephenson, T.J., Cotton, D.W.K., 1994. Fractal geometic analysis of colorectal polyps. J. Pathol. 172, 317-323.

Dairkee, S.H., Deng, S.H., Stampfer, M.R., Waldman, R.M., Smith, H.S., 1995. Selective cell culture of primary breast cancer. Cancer Res. 35, 2516-2519.

Fidler, I.J., Hart, I.R., 1982. Biological diversity in metastatic neoplasms: origins and implications. Science 217, 998-1003.

Franks, S.J., Byrne, H.M., Underwood, J.C., Lewis, C.E., 2005. Biological inferences from a mathematical model of comedo ductal carcinoma in situ of the breast. J. Theor. Biol. 232, 523-543.

Gatenby, R.A., Gawlinski, E.T., 1996. A reaction-diffusion model of cancer invasion. Cancer Res. 56, 5745-5753.

Gatenby, R.A., Gawlinski, E.T., 2003. The glycolytic phenotype in carcinogenesis and tumor invasion: insights through mathematical models. Cancer Res. 63, 3847-3854.

Gatenby, R.A., Maini, P.K., 2003. Mathematical oncology: cancer summed up. Nature 421, 321.

Gatenby, R.A., Gawlinski, E.T., Tangen, C., Flanigan, R.C., 2002. The possible role of post operative azotemia in enhanced survival of patients with metastatic renal cancer following cytoreductive nephrectomy. Cancer Res. 62, 5218-5222.

Greenspan, H.P., 1972. Models for the growth of a solid tumor by diffusion. Stud. Appl. Math. 51, 317-340.

Kelley, S.T., Manon, C., Buerk, D.G., Bauer, T.W., Fraker, D.L., 2002. Acidosis plus melphalan induces nitric oxide-mediated tumor regression in an isolated limb perfusion human melanoma xenograft model. Surgery 132, 252-258.

Kerangueven, F., Noguchi, T., Coulie, R.F., Allione, F., Wargniez, V., Simony-Lafontaine, J., Longy, M., Jacquemier, J., Sobol, H. Eisinger, F., Birnbaum, D., 1997. Genome-wide search for loss of heterozygosity shows extensive genetic diversity of human breast carcinomas. Cancer Res. 57, 5469-5474.
Kunkel, M., Reichert, T.E., Benz, P., Lehr, H., Jeong, J., Wieand, S., Bartenstein, P., Wagner, W., Whiteside, T., 2003. Overexpression of Glut-1 and increased glucose metabolism in tumors are associated with a poor prognosis in patients with oral squamous cell carcinoma. Cancer 97, 1015-1024.

Laird, A.K., 1964. Dynamics of tumour growth. Br. J. Cancer 18, $490-502$.

Martin, G.R., Jain, R.K., 1994. Noninvasive measurement of interstitial $\mathrm{pH}$ profiles in normal and neoplastic tissue using fluorescence ratio imaging microscopy. Cancer Res. 54, $5670-5674$

Martinez-Zaguilan, R., Seftor, E.A., Seftor, R.E., Chu, Y.W., Gillies, R.J., Hendrix, M.J., 1996. Acidic $\mathrm{pH}$ enhances the invasive behavior of human melanoma cells. Clin. Exp. Metastasis 14, 176-186.

McElwain, D.L.S., Morris, I.E., 1978. Apoptosis as a volume loss mechanism in mathematical models of solid tumor growth. Math. Biosci. 39, 147-157.

Morita, T., Nagaki, T., Fukuda, I., Okumura, K., 1992. Clastogenicity of low pH to various cultured mammalian cells. Mutat. Res. 268, 297-305.

Patel, A.A., Gawlinski, E.T., Lemieux, S.K., Gatenby, R.A., 2001. A cellular automaton model of early tumor growth and invasion. J. Theor. Biol. 213, 315-331.

Raghunand, N., Mahoney, B., van Sluis, R., Baggett, B., Gillies, R.J., 2001. Acute metabolic alkalosis enhances response of $\mathrm{C} 3 \mathrm{~h}$ mouse mammary tumors to the weak base mitoxantrone. Neoplasia 3, 227-235.

Vaupel, P., Kallinowski, F., Okunieff, P., 1989. Blood flow, oxygen and nutrient supply, and metabolic microenvironment of human tumors: a review. Cancer Res. 49, 6449-6465.

Warburg, O., 1930. The Metabolism of Tumours. Constable Press, London. 